\title{
Abortion practices undermining reformist laws - experts
}

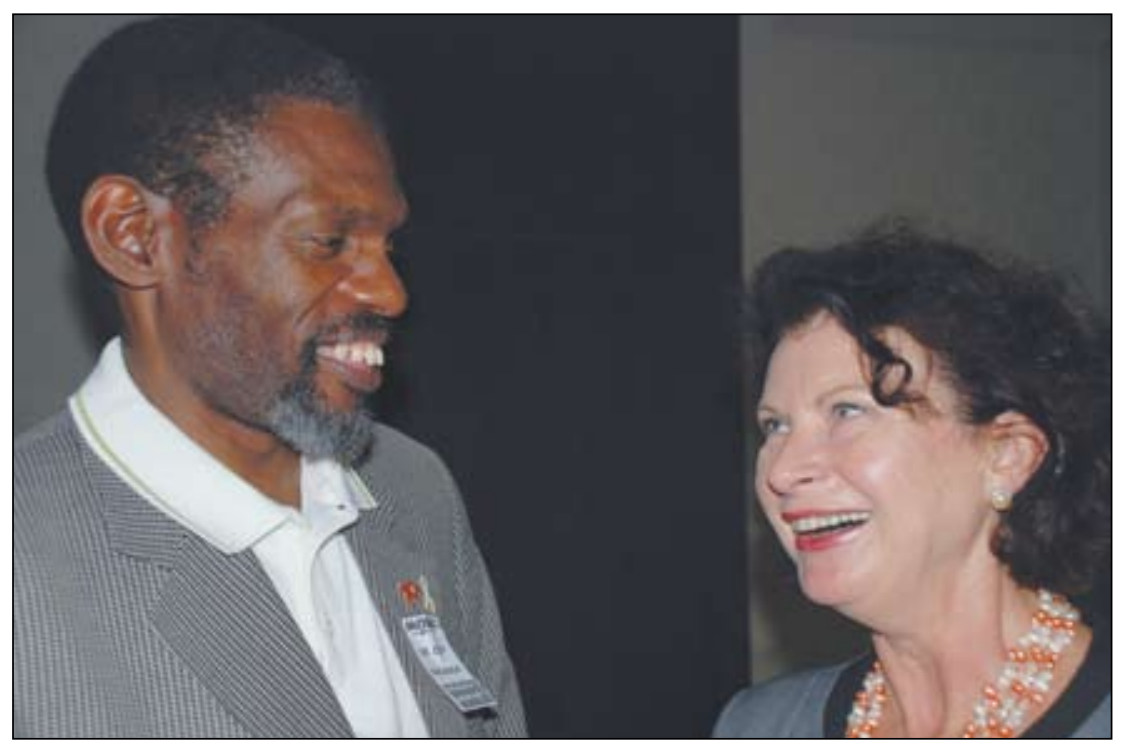

Dr Eddie Mhlanga, Cluster Manager of Maternal, Child and Women's Health in the national health department with Christélle Cronjé, executive director of Mosaic, a Western Cape-based NGO set up to help free society of abuse and domestic violence, particularly against women and children.

A combination of stigma, ignorance of reformist laws and health care facilities that offer abortion services being 'unidentifiable' mean that $43 \%$ of designated facilities today fail to provide first-trimester pregnancy termination services.

This emerged at a Dialogue on Safe Abortions, attended by many of the country's top experts on the subject and held at a Cape Town hotel last month.

Dr Eddie Mhlanga, Cluster Manager of Maternal, Child and Women's Health in the national health department, said it was 'very sad' that 3 years after the Choice on Termination of Pregnancy (CTOP) Act was liberally amended, only $57 \%$ of designated TOP facilities (including Marie Stopes private clinics) were actually functional.

The law was amended in 2008 to provide protocols and solid guidelines for designated facilities to enable any woman of childbearing age to request an abortion (without providing reasons before 13 weeks' gestation) and widen the field of TOP providers, thus addressing an ongoing local epidemic of dangerous and often fatal backstreet abortions. The 2005 - 2007 Saving Mothers Report shows an increase in the number of avoidable deaths from abortion from $4.7 \%$ to $4.9 \%$. This is the first time since the introduction of this report that an increase,
Picture: Chris Bateman

of conception for both first- and secondtrimester abortions (usually manual vacuum aspiration), creating unwieldy waiting lists that push many women into their second trimester anyway.

The CTOP Act allows for first-trimester abortions (up to 12 weeks' gestation) to be performed by trained registered nurses and midwives, whereas only doctors may perform second-trimester abortions.

\section{SA - double global norm for second-trimester abortions}

Mhlanga admitted that insufficient staff, conscientious objection and anti-abortion attitudes among staff and society, plus dysfunctional clinics and district hospitals, were aggravating factors and real barriers. The creative move by his department will hopefully reduce South Africa's unusually high number of requests for secondtrimester abortions (20 - 25\% higher than in most other countries where $10-13 \%$ is the norm). Insufficient support for providers of TOP also contributes to attrition of trained personnel.

maternal mortality has evidence suggesting the increase in deaths is due to unsafe abortions.

\section{White men in black suits decided for women}

The original Abortion and Sterilisation Act, 1975 (Act No. 2 of 1975), drawn up by '17 white men in black suits', as one delegate politely put it, allowed abortion only if the physical or mental health of the mother was threatened and/or there was evidence of fetal abnormality, rape or incest. The pregnant woman also needed a psychiatric examination and the signature of the relevant hospital's medical superintendent to secure a legal abortion.

Mhlanga revealed that within days of the 23 March Dialogue, he would seek a go-ahead from the National Health Council (previously MINMEC) to provide both mifepristone and misoprostol tablets to women requesting first-trimester abortions. He explained that this was to increase accessibility to safe State TOP services by hopefully reducing the pressure of second-trimester abortions in the context of low hospital bed and theatre availability. This was intended to minimise the practice of designated State facilities offering only mifepristone, followed by the surgical removal of the products
The creative move by his department will hopefully reduce South Africa's unusually high number of requests for secondtrimester abortions (20 - 25\%

higher than in most other countries where $10-13 \%$ is the norm). Insufficient support for providers of TOP also contributes to attrition of trained personnel.

Dr Marijke Alblas, a consultant at Tygerberg Hospital, TOP trainer and board member of Mosaic (a training service and healing centre for women which organised the 23 March Dialogue), said secondtrimester TOP figures at one youth clinic she helped start in Khayelitsha township were over $50 \%$. She said that although more research was urgently needed on the reasons why women regularly presented late, anecdotal evidence was that those who missed their regular contraceptive injection failed to notice the absence of their periods and therefore advancing pregnancies. However, multiple and complex interrelated factors influenced the timing of seeking an abortion, including indecision due to 


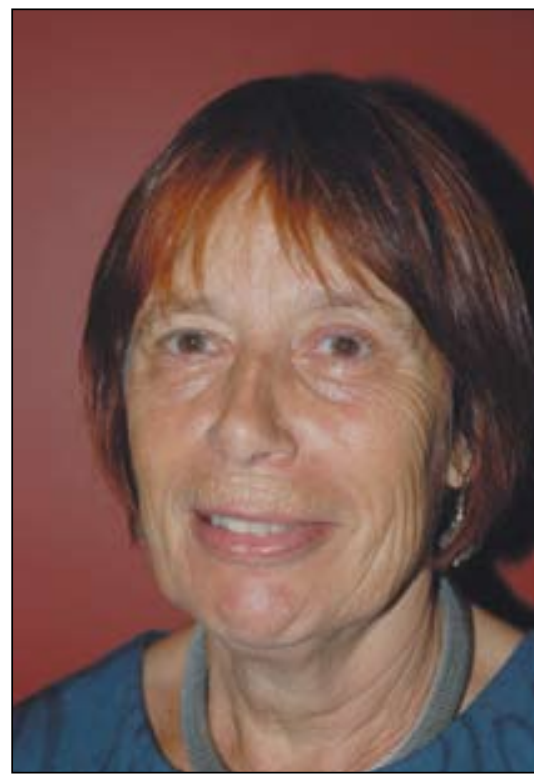

Dr Marijke Alblas, a consultant who helps women with second-trimester abortions wherever functional facilities exist in the Western Cape.

Picture: Chris Bateman

changed personal circumstances and health service-related barriers. Her experience in the Western Cape, one of the most efficient and progressive provinces when it comes to TOP, was that most hospitals did not perform surgical abortions. It was 'easier just to give the tablets and let the woman do the dirty job herself'. Her experience was that nurses usually gave patients only misoprostol (after which $72 \%$ of women usually aborted with 24 hours), whereas the combination of misoprostol and mifepristone increased this to a success rate of $97 \%$.

\section{Nurses gate-keeping abortion services}

Referring to Dr Mhlanga's admission about the aggravating systemic factors that created barriers, she said that even at Tygerberg Hospital the system was patient unfriendly. A woman first had to wait for a booking clerk, then visit a doctor for a sonar, then see a social worker (whom she claimed generally (and illegally) decided for the patient whether they should terminate).
'It's mostly socio-economic reasons and the social worker generally decides whether they can afford another child - and over 16 weeks [the nurses] generally refuse to book them - basically they make their own rules', she added.

\section{'It's mostly socio-economic} reasons and the social worker generally decides whether they can afford another child - and

over 16 weeks [the nurses] generally refuse to book them basically they make their own rules,' she added.

In spite of World Health Organization (WHO) guidelines, few provinces had protocols on second-trimester abortion and there was 'very little support for us doing second-trimester abortions. Nurses do it [first-trimester abortions] for a while but they stop because there's no support. Every hospital I've worked in has a small separate unit where it's done, off in a corner somewhere. It's not integrated and you're very much on your own.'

At Somerset Hospital women waited up to 6 weeks before they could be helped, meaning that those who arrived at 13 weeks were 'pushed to 18 weeks' before they could be assisted, with all the attendant risks and stigma. Dr Alblas said she was often judgmentally asked 'how I can do this?'

'I think a child has a right to be born wanted. There are enough unwanted children in the world,' she said, adding that because there were so few doctors and such a high demand, many women ended up aborting at home after taking misoprostol.

\section{'Do you have any idea what it} means to expel at home or on your way to hospital? This should not be allowed but it's been going on for years - there are simply too many procedures to get through in one day.'
'Do you have any idea what it means to expel at home or on your way to hospital? This should not be allowed but it's been going on for years - there are simply too many procedures to get through in one day.'

Dr Karen Trueman, a pivotal local member of Ipas, the only South African NGO focusing exclusively on abortion care, said more focus on information (including easy identification of hospitals designated as TOP sites), contraception and abortion services would alleviate much of the secondtrimester abortion problem.

'And if you catch one of these [backstreet abortion] advertising charlatans, take them to court! The law provides for a stiff fine and/or jail for a maximum of ten years,' she stressed. Sister Sandra Mabila, Mosaic's clinical manager, said that in the absence of easy access to TOP services, unsafe backstreet abortionists were 'mushrooming visibly'.

\section{Mabila challenged 'pro-choice or pro-lifers' to 'take one of those backstreet pamphlets and do something about those people - instead of targeting those of us trying to save women's lives'.}

'I took 50 pamphlets from a guy the other day. This while our TOP services are collapsing due to a lack of both political and managerial support,' she added. Mabila challenged 'prochoice or pro-lifers' to 'take one of those backstreet pamphlets and do something about those people - instead of targeting those of us trying to save women's lives'.

Trueman said that anyone who prevented the lawful termination of a pregnancy or obstructed access to a TOP facility was guilty of an offence. She estimates that the CTOP Act (as amended) is currently being implemented to about $30 \%$ of its potential efficacy.

\section{Chris Bateman}

